\title{
The Linear Potential Propagator via Wave Function Expansion
}

\author{
Antônio B. Nassar, ${ }^{(1,2)}$ José M. F. Bassalo, ${ }^{(2)}$ Paulo T. S. Alencar, ${ }^{(2)}$ \\ José L. M. Lopes, ${ }^{(2)}$ José I. F. de Oliveira, ${ }^{(3)}$ and Mauro S. D. Cattani ${ }^{(4)}$ \\ ${ }^{(1)}$ Extension Program-Department of Sciences, University of California, Los Angeles, California 90024, USA \\ (2) Departamento de Física da UFPA, 66075-900, Belém, Pará, Brazil \\ ${ }^{(3)}$ Departamento de Física da UNAMA, 66050-000, Belém, Pará, Brazil \\ ${ }^{(4)}$ Instituto de Física da USP, C.P. 66318, 05315-970, São Paulo, SP, Brazil
}

Received on 3 June, 2002

\begin{abstract}
We evaluate the quantum propagator for the motion of a particle in a linear potential via a recently developped formalism [A.B. Nassar et al., Phys. Rev. E56, 1230, (1997)]. In this formalism, the propagator comes about as a type of expansion of the wave function over the space of the initial velocities.
\end{abstract}

\section{Introduction}

The quantum propagator for the motion of a particle in a linear potential has been recently discussed by several authors[1-4] using different techniques. This arsenal of techniques has an immediate pedagogical appeal and can be valuable in some future calculation which may require the use of a nonstandard approach.

Here we present an alternative approach for the evaluation of the linear potential propagator via a new formalism developed by Nassar et al.[5] In this formalism the central result is

$$
K\left(x, t ; x_{o}, t_{o}\right)=(m / 2 \pi \hbar) \times \int_{-\infty}^{+\infty} d v_{o} F\left(x, t, x_{o}, t_{o}, v_{o}\right) e^{\frac{i}{\hbar} S_{\text {quantum }}\left(x, t, x_{o}, t_{o}, v_{o}\right)}
$$

where

$$
\begin{gathered}
F\left(x, t, x_{o}, t_{o}, v_{o}\right)=\left(\frac{a(t)}{a_{o}}\right)^{-1 / 2} \\
\times \exp \left[\left(\frac{i m \dot{a}(t)}{2 \hbar a(t)}-\frac{1}{4 a^{2}(t)}\right)[x-X(t)]^{2}+\frac{i m \dot{X}(t)}{\hbar}[x-X(t)]\right] \\
S_{\text {quantum }}\left(x, t, x_{o}, t_{o}, v_{o}\right)=\int_{o}^{t} d t^{\prime}\left(\frac{1}{2} m \dot{X}^{2}\left(t^{\prime}\right)-V\left[X\left(t^{\prime}\right)\right]-\frac{\hbar^{2}}{4 m a^{2}\left(t^{\prime}\right)}\right),
\end{gathered}
$$

$X(t)$ is the solution to the classical equation

$$
\ddot{X}=-\frac{1}{m} V^{\prime}[X(t), t],
$$

and $a(t)$ is the solution to the quantum equation

$$
\ddot{a}+\left(\frac{1}{m} V^{\prime \prime}[X(t), t]\right) a=\frac{\hbar^{2}}{4 m^{2} a^{3}} .
$$


The terms on the right-hand side of Equations (4) and (5) are due the presence of the classical and the quantum potential, respectively.

Equation (1) provides the propagator as a type of expansion of the wave function over the space of the initial velocities. It is worth noticing the presence of the quantum potential in the quantum action (Equation (3)) and in the expansion coefficient (Equation (2)) through the function $a(t)$.

\section{Linear Potential}

In what follows, we calculate in some detail the quantum propagator for the linear potential $V=-f x$ through the formalism given by Equations $(1,2,3)$, therefore demonstrating its validity and usefulness as a new tool for future investigations.

First, we find the solutions to Equations (4) and (5), subject to the initial conditions

$$
X(0)=x_{o}, \quad \dot{X}_{o}=v_{o} ; \quad a(0)=a_{o}, \quad \dot{a}(0)=0,
$$

to be

$$
\begin{gathered}
X(t)=\frac{f}{2 m} t^{2}+v_{o} t+x_{o}, \\
a(t)=a_{o} \sqrt{1+(t / \tau)^{2}},
\end{gathered}
$$

where $\tau=\frac{2 m a_{o}^{2}}{\hbar}$.

Equations (7) and (8) allow us to carry out in full the arguments of the exponent of Equations $(1,2,3)$ as follows. The first and the second arguments of Equation (2) can be expanded, respectively, as

$$
\begin{gathered}
\left(\frac{i m \dot{a}(t)}{2 \hbar a(t)}-\frac{1}{4 a^{2}(t)}\right)[x-X(t)]^{2}=\frac{1}{4 a_{o}^{2}\left[1+(t / \tau)^{2}\right]}\left[\frac{i t \hbar}{2 a_{o}^{2} m}-1\right] \\
\times\left[\left(x-x_{o}\right)^{2}+\frac{f^{2} t^{4}}{4 m^{2}}+v_{o}^{2} t^{2}+\frac{f t^{2}}{m} v_{o} t-\left(x-x_{o}\right)\left(\frac{f t^{2}}{m}-2 v_{o} t\right)\right] \\
\frac{i m \dot{X}(t)}{\hbar}[x-X(t)]=\frac{i m}{\hbar}\left[\left(x-x_{o}\right) v_{o}-v_{o}^{2} t\right. \\
+\left(x-x_{o}\right)\left[\frac{f t}{m}-\frac{f^{2} t^{3}}{2 m^{2}}-\frac{3 f t^{2}}{2 m}\right] .
\end{gathered}
$$

Likewise, the quantum action (Equation (3)) can be expressed as

$$
\begin{gathered}
\int_{o}^{t} d t^{\prime}\left(\frac{1}{2} m \dot{X}^{2}\left(t^{\prime}\right)+f X\left(t^{\prime}\right)-\frac{\hbar^{2}}{4 m a^{2}\left(t^{\prime}\right)}\right)= \\
=\frac{1}{2} m v_{o}^{2}+f x_{o}+\frac{f^{2} t^{2}}{m}+2 f t v_{o}-\frac{\hbar^{2}}{4 m a_{o}^{2}\left[1+(t / \tau)^{2}\right]} .
\end{gathered}
$$

The propagator in Equation (1) is of the general form

$$
\begin{gathered}
K=D(t) \int_{-\infty}^{+\infty} d v_{o} \exp \left(-A(t) v_{o}^{2}+B(t) v_{o}+C(t)\right)= \\
=D(t) \sqrt{\frac{\pi}{A(t)}} \exp \left(\frac{B^{2}(t)}{4 A(t)}+C(t)\right),
\end{gathered}
$$

where the coefficients $A(t), B(t), C(t)$ and $D(t)$ are, respectively:

$$
\begin{gathered}
A(t)=-\left[\frac{t^{2}}{4 a^{2}}+\frac{i m t}{\hbar\left[1+(t / \tau)^{2}\right]}\right], \\
B(t)=\left[\frac{t}{2 a^{2}}+\frac{i m}{\hbar\left[1+(t / \tau)^{2}\right]}\right]\left[\left(x-x_{o}\right)-\frac{f t^{2}}{2 m}\right],
\end{gathered}
$$




$$
\begin{gathered}
C(t)=\frac{i m}{2 \hbar} v_{o}^{2} t-\frac{i}{2} \tan ^{-1}(t / \tau)+\frac{i}{\hbar} f x_{o} t+\frac{i}{\hbar} \frac{f^{2} t^{3}}{3 m}+\frac{i}{\hbar} v_{o} f t^{2}, \\
D(t)=\frac{m}{2 \pi \hbar}\left[\frac{a(t)}{a_{o}}\right]^{-1 / 2} .
\end{gathered}
$$

The terms in $\left(x-x_{o}\right)^{2},\left(x+x_{o}\right)$, and independent of $x$ and $x_{o}$ are, respectively,

$$
\begin{gathered}
\frac{i}{\hbar} \frac{m}{2 t}\left(x-x_{o}\right)^{2}, \\
\frac{i}{2 \hbar} f t\left(x+x_{o}\right), \\
-\frac{i}{\hbar} \frac{f^{2} t^{3}}{24 m}-\frac{i}{2} \tan ^{-1}(t / \tau) .
\end{gathered}
$$

This allows us to write

$$
K\left(x, t ; x_{o}, 0\right)=\left(\frac{m}{2 \pi i \hbar t}\right)^{1 / 2} \exp \left[\frac{i}{\hbar}\left[\frac{m}{2 t}\left(x-x_{o}\right)^{2}+\frac{f t}{2}\left(x+x_{o}\right)-\frac{f^{2} t^{3}}{24 m}\right]\right],
$$

which is the result found in Refs. [1-4].

We have presented a new approach for the evaluation of the linear potential propagator. In this formalism, the propagator comes about as a type of expansion of the wave function over the space of the initial velocities. This new approach has a pedagogical appeal and poses an alternative route for future research investigations.

\section{References}

[1] B. R. Holstein, Am. J. Phys. 65, 414 (1997).
[2] G. P. Arrighini, N. L. Durante and C. Guidotti, Am. J. Phys. 64, 1036 (1996).

[3] R. W. Robinett, Am. J. Phys. 64, 803 (1996).

[4] L. S. Brown and Y. Zhang, Am. J. Phys. 62, 806 (1994).

[5] A. B. Nassar, J. M. F. Bassalo, P. T. S. Alencar, L. S. G. Cancela and M. Cattani, Phys. Rev. E56, 1230 (1997). 